\section{Unintentional percutaneous aspiration
of a pleural hydatid cyst}

Mohamed Ali Al Karawi, Abdel Rahman El Shiekh Mohamed, Babiker Omer El Tayeb, Mohamed Ismail Yasawy

\begin{abstract}
A Saudi man underwent repeated percutaneous aspiration of a pleural effusion before a pleural biopsy showed hydatid disease. Subsequent investigations confirmed hydatid disease in the pleura and liver. The patient underwent surgical evacuation and irrigation of the pleural cavity followed by medical treatment for the liver cyst with albendazole, with complete recovery and disappearance of the liver cyst.
\end{abstract}

\section{Case report}

A 59 year old Saudi man was admitted to hospital with recurrent right sided pleural effusion. He had been well until the age of 52, when he first developed a right sided exudative pleural effusion. He was a heavy cigarette smoker.

After extensive investigations elsewhere to exclude malignancy, he was treated empirically with antituberculous drugs for one year, though there was no bacteriological confirmation of tuberculosis. He was admitted to hospital repeatedly with massive right sided pleural effusions needing therapeutic and diagnostic tapping. The aspirates were always interpreted as non-specific effusion, though they were not examined specifically for Echinococcus granulosus. When the effusion persisted pleural biopsy was performed; the biopsy specimen showed segments of laminated hydatid membrane, a few hooklets, and many degnerated scolices (fig 1). Computed tomography of the abdomen and chest (fig 2)

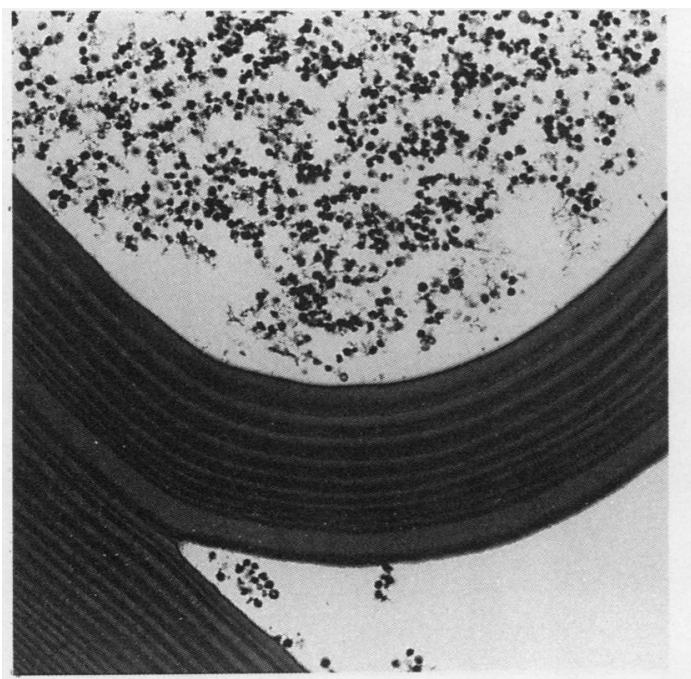

showed a liver cyst and a cystic lesion within the pleural cavity associated with a large pleural effusion. Magnetic resonance imaging of the chest and upper abdomen gave the same results. The hydatid antibody titre was raised at $\mathbf{1 / 8 1 9 2}$ (indirect haemagglutination test).

Eventually the patient had a right thoracotomy to evacuate the pleural cyst. Intraoperatively there was thickening of the parietal and visceral pleura, forming a pericyst with multiple daughter cysts in the pleural cavity.

The pleural cysts were enucleated and the pleural cavity was instilled with $0.5 \%$ silver nitrate. There was no communication through the diaphragm with the hepatic cyst and the surgeon did not attempt to remove the liver cyst because of the patient's general condition. The patient was treated with albendazole $400 \mathrm{mg}$ twice daily for four weeks followed by two weeks without treatment and a further seven courses of albendazole over one year.

Follow up with computed tomography and ultrasound showed regression and almost complete disappearance of the liver cyst and serial hydatid antibody titres showed a progressive fall from $1 / 8192$ to $1 / 32$ (negative) after one year. Forty two months after treatment the patient remained well with no recurrence of the pleural effusion.

\section{Discussion}

The incidence of human echinococcosis in Saudi Arabia has not been ascertained but the disease is not uncommon. ${ }^{1}$ In areas where hydatid disease is common the diagnosis should be considered before surgery or aspiration of any cyst or unexplained persistent pleural effusion..$^{2-5}$ Percutaneous aspiration of a suspected hydatid cyst is not recommended generally because of the risk of an allergic reaction, which can be serious with asthma or systemic anaphylaxis, and because of the danger of spread of the disease by spillage of the cyst's contents. ${ }^{56}$ Aspiration of a hydatid cyst is not necessarily followed by an anaphylactic reaction, however, as shown by the present case and others. We believe that an anaphylac-

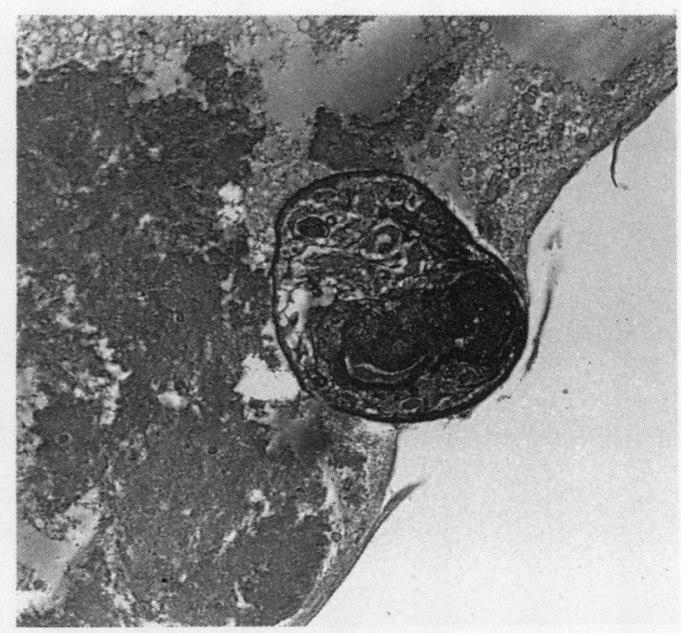

Figure 1 Pleural biopsy specimen showing (a) hydatid and (b) scolices.

Reprint requests to: Dr Mohamed

Accepted 17 April 1991 


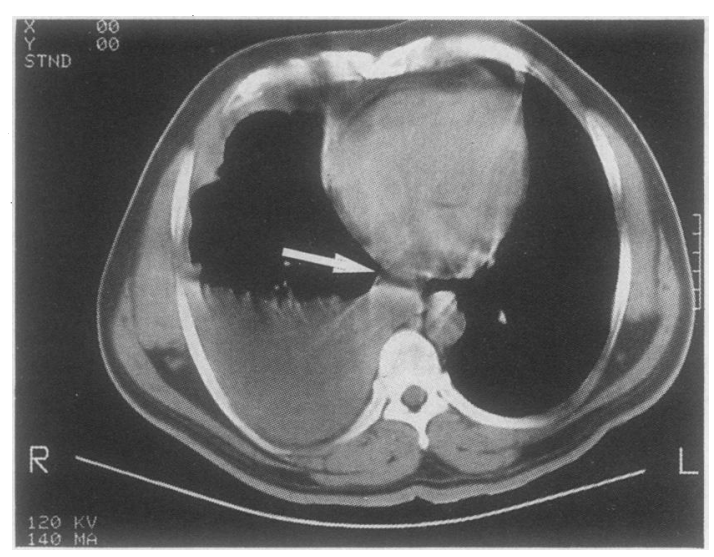

Figure 2 Computed tomogram showing the hydatid cyst (arrow) in liver and pleural effusion.

tic reaction occurs only if there is a direct contact between the fluid from the ruptured cyst and the circulation or if the patient is allergic to hydatid fluid.

Reports of unintended and intended aspiration of pulmonary and hepatic hydatid cysts have accumulated. ${ }^{5-9}$ McCorkell $^{6}$ reported three patients in whom the diagnosis of hydatid disease was made by percutaneous thoracic aspiration without deleterious effects. Brett et al reported on 13 patients who had percutaneous aspiration and drainage of hydatid cysts in the liver and concluded that percutaneous aspiration can be an alternative to surgery in selected cases. ${ }^{9}$

Non-surgical management of hydatid disease by percutaneous aspiration of echinococcus has been found to be safe and effective in different series and, unlike surgery, ${ }^{5-9}$ has not been associated with major complications. When a hydatid liver cyst ruptures into the biliary tree to cause obstructive jaundice nonsurgical endoscopic management may be effective. ${ }^{10}$
We have reported on the effectiveness of albendazole treatment in 22 patients, ${ }^{11}$ who had hydatid disease affecting mainly the liver though two had pulmonary cysts, and Morris also found albendazole to be effective in 24 patients with hydatid cysts. ${ }^{12}$

Although percutaneous aspiration of echinococcal cysts of the lungs and liver has been performed without serious complications, ${ }^{6-9}$ we believe this to be the first report of an unintentional percutaneous aspiration of a pleural echinococcal cyst. Neither computed tomography nor magnetic resonance imaging showed the lung parenchyma to be affected.

1 Malaika S, Attayeb A, Sulaimani S, Reddy J. Human echinococcosis in Saudi Arabia. Saudi Med J 1981;2: 77-84.

2 Hankins J, Werner D, Kobout E. Surgical treatment of ruptured and unruptured hydatid cysts of the lung. Ann Surg 1968;31167:336-41.

3 Al Kraida A, Alam M, Qazi S, Al Qasabi Q, Bashier A Hydatid disease of the liver in Riyadh. Ann Saudi Med 1988;8:117-21.

4 Kagan IG. Serodiagnosis of parasitic disease. In: Rose NR, Friedman H, eds. Manual of clinical immunology. American Society for Microbiology, 1980:580.

5 Stampfel G. Anaphylaktoide reaktion als seltene Komplikation der Lungen-feinnadelpunktion. Radiologie 1982;22:329-30.

6 McCorkell SJ. Unintentional percutaneous aspiration of pulmonary echinococcal cysts. AJR 1984;143:123-26.

7 Muller PR, Dawson SL, Ferrucci JT, Nordi GL. Hepatic echinococcal cyst: successful percutaneous drainage. Radiology 1985;155:627-8.

8 Al Karawi MA, Hanid MA, Mohamed AE. Transpapillary endoscopic nasobiliary drainage of a hepatic abscess presumably hydatid in origin. Saudi Med J 1988;9: 208-13.

9 Brett PM, Fond A, Bretagnolle M, et al. Percutaneous and drainage of hydatid cysts in the liver. Radiology 1988;168:617-20.

10 Al Karawi MA, Mohamed AE, Yasawy MI, Haleem A. Nonsurgical endoscopic transpapillary treatment of ruptured echinococcus liver cyst obstructing the biliary tree. Endoscopy 1987;2:81-3.

11 Al Karawi MA, Mohamed AE, Yasawy MI. Advances in diagnosis and management of hydatid disease. HepatoGastroenterology 1990;37:327-31.

12 Morris DL. The use of albendazole in human hydatid disease. Ann Trop Med Parasitol 1984;78:204-5. 